\title{
Periodic chain of disks in a magnetic field: bulk states and edge states
}

\author{
E. N. Grishanov ${ }^{1}$, D. A. Eremin ${ }^{1}$, D. A. Ivanov ${ }^{1}$, \\ I. Yu. Popov ${ }^{2}$, P. I. Smirnov ${ }^{2}$ \\ ${ }^{1}$ Department of Mathematics and IT, \\ Ogarev Mordovia State University \\ Bolshevistskaya Str. 68, Saransk, Russia \\ ${ }^{2}$ Department of Higher Mathematics, ITMO University, \\ Kroverkskiy pr. 49, St. Petersburg, 197101, Russia \\ popov1955@gmail.com
}

An explicitly solvable model for periodic chain of coupled disks in orthogonal magnetic field is considered. The spectrum for the Hamiltonian is compared with the spectrum for the corresponding chain of circles. These models are used for the comparison of the bulk and edge states. It is found that for some range of the magnetic field values the lowest band for the circles system lies below the spectrum for the corresponding disks system, i.e. the edge band is below and is separated from the lowest bulk band.

Keywords: nanostructure; magnetic field; explicitly solvable model.

Received: 1 September 2015

\section{Introduction}

The difference between surface and bulk conductivities for materials and nanostructures is a subject of numerous experimental and theoretical investigations due to intriguing prospects (high-temperature superconductivity, topological insulator, etc.). In many cases, low-dimensional nanostructures have unusual electronic properties, see, e.g., results of recent experiments with highly ordered organometallic nanoribbons, whose intrinsic (defect-free) conductivity is found to be three orders of magnitude higher than that of macroscopic crystals [1]. It should be mentioned that in many cases there is no adequate mathematical model of the corresponding nanosystem, and the theoretical description is complex. Our goal is to construct a mathematical model for chain type periodic nanostructure and to describe its spectrum. Ideally, we would like to have a band (preferably, the lowest band) for the surface (edge) states be in the gap (or below the first band) for the bulk states. The corresponding system is described in the present paper. This case is, in some sense, similar to a topological insulator (TI). TIs are electronic materials that have a bulk band gap like an ordinary insulator, but have protected conducting states on their edge or surface [2-5]. We have similar phenomenon for surface state protection as in TI, although the physical nature is different.

We consider a periodic system of coupled disks as a basic geometry. As for the problem of edge states, our consideration is in the framework of a model of quantum graphs (periodic system of coupled circles). This model was used intensively in previous publications (starting from works $[6,7]$ ) due to its advantages: in one way, it is quasi-one-dimensional and rather simple, however, despite this apparent simplicity, it preserves the properties of the corresponding physical system, particularly, its spectral properties [8-13]. As for the problem of bulk states, we deal with the model of quantum resonators coupled through point-like window. This model 
is based on the theory of self-adjoint extensions of symmetric operators (see, e.g., [21]). We compare the spectra of the Landau operators for these two models and observe that for some values of the magnetic field, one has an edge state band below the first bulk band. The structure of the paper is rather natural. We describe, consequently, the models for chain of circles and for chain of disks, and then compare the corresponding spectra.

\section{Chain of circles}

Consider an infinite chain of circles $C_{n}, n=1,2, \ldots$ of radii $a$. The state space is $\mathcal{H}=\bigoplus_{n=1}^{+\infty} L^{2}\left(C_{n}\right)$. To construct the Hamiltonian of the charge particle for the chain of coupled circles, we start from self-adjoint operator $H_{c c}, H_{c c}=\bigoplus_{n=1}^{+\infty} H_{c}^{n}$. Here $H_{c}^{n}$ is the Landau operator for the circle $C_{n}$. Using the standard polar coordinates $(r, \varphi)$ on the circle, one can represent the Hamiltonian in the following form:

$$
H_{c}^{n}=\frac{\hbar^{2}}{2 m a^{2}}\left(-\imath \frac{\partial}{\partial \varphi}+\frac{\Phi}{\Phi_{0}}\right)^{2}
$$

Here, $m$ is an electron mass, $\Phi=\pi a^{2} B$ is a magnetic flux, $\Phi_{0}=\frac{\hbar}{2 c}$ is the quantum of the magnetic flux. We use the unit system such that the factor $\frac{\hbar^{2}}{2 m a^{2}}$ is equal to 1 (i.e. $\hbar=1, m=1 / 2, a=1)$.

A rigorous mathematical model of our system of coupled circles is constructed by a conventional way by use of the operator extensions theory (see, e.g., [14-19]). We assume that the contacts are located at the opposite points $\left(a, \varphi_{1}\right)$ and $\left(a, \varphi_{2}\right)$, where $\varphi_{1}=0, \varphi_{2}=\pi$ (see Fig. 1). We restrict the initial operator $H_{c c}$ on the set $D_{0}=\bigcup_{n=1}^{+\infty}\left\{f \in D\left(H_{c c}\right): f\left(q_{n}\right)=0\right\}$, where $q_{n}$ is the contact point. The model Hamiltonian is given by a self-adjoint extension of this symmetric operator. We choose the extension corresponding to the following boundary conditions:

$$
\left\{\begin{array}{l}
\psi^{n+1}\left(\varphi_{2}^{n+1}\right)=e^{\imath q l} \psi^{n}\left(\varphi_{2}^{n}\right) \\
\psi^{\prime n+1}\left(\varphi_{2}^{n+1}+0\right)-\psi^{\prime n+1}\left(\varphi_{2}^{n+1}-0\right)=e^{\imath q l}\left[\psi^{\prime n}\left(\varphi_{2}^{n}+0\right)-\psi^{\prime n}\left(\varphi_{2}^{n}-0\right)\right] \\
\psi^{n+1}\left(\varphi_{2}^{n+1}\right)=\psi^{n}\left(\varphi_{1}^{n}\right)= \\
\beta\left[\psi^{\prime n+1}\left(\varphi_{2}^{n+1}+0\right)-\psi^{\prime n+1}\left(\varphi_{2}^{n+1}-0\right)+\psi^{\prime n}\left(\varphi_{1}^{n}+0\right)-\psi^{\prime n}\left(\varphi_{1}^{n}-0\right)\right]
\end{array}\right.
$$

where $l=2 a=2, q$ is the quasi-momentum, $\beta$ is the coupling parameter for the contact points.

Taking into account the expression for the electron wave function $\psi(\varphi)$ :

$$
\psi(\varphi)=\alpha_{1} G\left(\varphi, \varphi_{1}, E\right)+\alpha_{2} G\left(\varphi, \varphi_{2}, E\right),
$$

and (1), we obtain the following dispersion equation:

$$
4 Q_{21} \cos (q l)+\beta^{-1} \operatorname{det} Q-2 \operatorname{Tr} Q=0,
$$




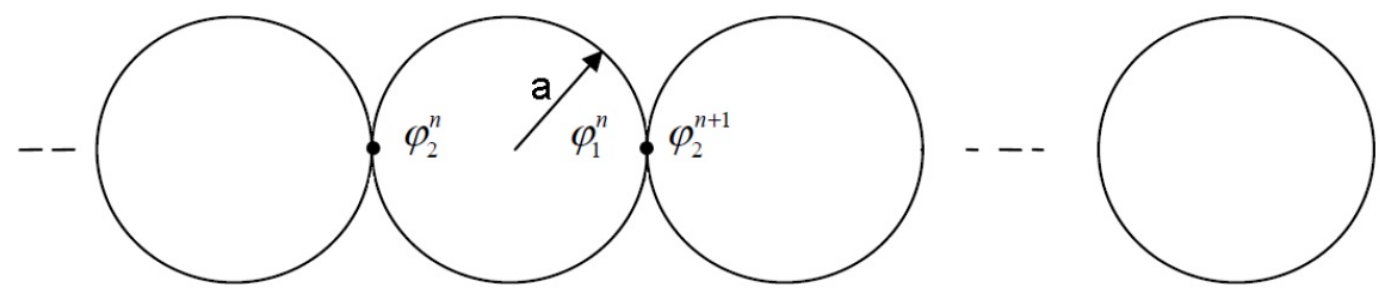

FIG. 1. Chain of circles (disks)

where $Q$ is the Krein $Q$-matrix:

$$
\begin{aligned}
& Q_{11}(E)=Q_{22}(E)=\frac{1}{k r}\left[\frac{\sin \pi k r \cos \pi k r}{\sin ^{2} \pi \eta-\sin ^{2} \pi k r}\right], \\
& Q_{12}(E)=Q_{21}(E)=\frac{1}{k r}\left[\frac{\sin \pi k r \cos \pi \eta}{\sin ^{2} \pi \eta-\sin ^{2} \pi k r}\right] .
\end{aligned}
$$

Solutions of (1) for various quasi-momenta give us the spectral bands.

\section{Chain of disks}

In this section, we consider a system with similar geometrical structure but circles in the chain are replaced by disks of the same radius $D_{n}=\{(r, \varphi): r \leq a\}$. Here, $r, \varphi$ are the standard polar coordinates. The initial Hamiltonian for our procedure is $H_{c d}=\bigoplus_{n=1}^{+\infty} H_{d}^{n}$, where $H_{d}^{n}$ is the Landau operator for the disk $D_{n}$ with the Dirichlet boundary conditions:

$$
H_{d}=-\frac{1}{2 m}\left(\imath \hbar \nabla-\frac{e}{2 c} B \times \mathbf{r}\right)^{2} .
$$

We introduce the following notations:

$$
\omega=\frac{|e B|}{c m}, \mu=\left(\frac{\hbar c}{|e B|}\right)^{1 / 2}, x_{0}=\frac{a}{2 \mu^{2}} .
$$

An eigenfunction of the operator $H_{c d}$ with the Dirichlet boundary condition is as follows:

$$
\psi(r, \varphi)=\left(\frac{m \omega}{2 \pi \hbar c_{l n}}\right)^{1 / 2} \frac{r^{|l|}}{\left(2 \mu^{2}\right)^{|l| / 2}} \exp \left(\imath l \varphi-\frac{r^{2}}{4 \pi \mu^{2}}\right) \Phi\left(\varepsilon_{l n},|l|+1, \frac{r^{2}}{2 \mu^{2}}\right) .
$$

We denote by $E_{l n}, l=0, \pm 1, \pm 2, \ldots$ the corresponding eigenvalue:

$$
E_{l n}=\hbar \omega\left(\frac{l+|l|+1}{2}-\varepsilon_{l n}\right)
$$

where $\varepsilon_{l n}$ is the $n$-th root of the equation $\Phi\left(\varepsilon,|l|+1, x_{0}\right)=0, \Phi$ is the Kummer function. Here, $c_{l n}$ is the normalization constant:

$$
c_{l n}=\int_{0}^{x_{0}} \exp (-x) x^{|l|} \Phi^{2}\left(\varepsilon_{l n},|l|+1, x\right) d x
$$


The Green function for a single disk has the following form [20]:

$$
\begin{aligned}
G\left(r, \varphi, r^{\prime}, \varphi^{\prime} ; E\right)=\frac{m \omega}{2 \pi \hbar} \exp \left(-\frac{r^{2}+r^{\prime 2}}{4 \mu^{2}}\right) \sum_{l=-\infty}^{+\infty} \frac{\left(r r^{\prime}\right)^{|l|}}{\left(2 \mu^{2}\right)^{|l|}} \exp \left(\imath l\left(\varphi-\varphi^{\prime}\right)\right) \times \\
\sum_{n=1}^{+\infty} \frac{\Phi\left(\varepsilon_{l n},|l|+1, \frac{r^{2}}{2 \mu^{2}}\right) \Phi\left(\varepsilon_{l n},|l|+1, \frac{r^{\prime 2}}{2 \mu^{2}}\right)}{c_{l n}\left(E_{l n}-E\right)} .
\end{aligned}
$$

To construct the model for coupled disks, we use the operator extensions theory model, more precisely, the model of zero-width slit [21]. This model faces difficulties for the Dirichlet boundary condition. In this case, one has to extend the initial Hilbert space to the Pontryagin space with indefinite metrics. The mathematical structure of this model has been described previously, e.g., in [22]. Here, we present the resulting formulas for our case.

The wave function for the $j$-th disk has the form:

$$
\psi_{j}(r, \varphi)=\left.\alpha_{1}^{j} \frac{\partial G\left(r, \varphi ; r^{\prime}, \varphi^{\prime} ; E\right)}{\partial r^{\prime}}\right|_{r^{\prime}=1, \varphi^{\prime}=0}+\left.\alpha_{2}^{j} \frac{\partial G\left(r, \varphi ; r^{\prime}, \varphi^{\prime} ; E\right)}{\partial r^{\prime}}\right|_{r^{\prime}=1, \varphi^{\prime}=\pi} .
$$

The asymptotics of this function near the chosen (contact) points $(r, \varphi)=(1,0)$, $(r, \varphi)=(1, \pi)$ contain a singular term. To construct a self-adjoint extension in the Pontryagin space (i.e. the model Hamiltonian), one should introduce a linear relation between the main singular and regular terms of the asymptotics from the both sides of the contact points (i.e. in neighbor disks).

We assume the existence of the $\delta$-potentials at the contact points. This gives us a rule for selection of the self-adjoint extension from the family of extensions.

As for the chain, we should take into account the periodicity. This can be made using the Bloch's theory (similarly to the case of circles) or by the transfer-matrix technique (see, e.g. [14]). The transfer-matrix $M$ is determined as follows:

$$
\left(\begin{array}{c}
a_{j+1} \\
b_{j+1}
\end{array}\right)=M\left(\begin{array}{c}
a_{j} \\
b_{j}
\end{array}\right)
$$

where:

$$
\left\{\begin{array}{l}
a_{j}=\alpha_{2}^{j}, \\
a_{j+1}=\alpha_{2}^{j+1}, \\
b_{j}=q_{12} \alpha_{2}^{j}+q_{11} \alpha_{1}^{j}, \\
b_{j+1}=q_{12} \alpha_{2}^{j+1}+\alpha_{1}^{j+1} q_{11} .
\end{array}\right.
$$

In this formula, the following notation is used:

$$
\begin{gathered}
q_{11}=\left.\frac{\partial}{\partial r}\left(\left.\frac{\partial G\left(r, \varphi ; r^{\prime}, \varphi^{\prime} ; E\right)}{\partial r^{\prime}}\right|_{r^{\prime}=1, \varphi^{\prime}=0}-\left.\frac{\partial G\left(r, \varphi ; r^{\prime}, \varphi^{\prime} ; E_{0}\right)}{\partial r^{\prime}}\right|_{r^{\prime}=1, \varphi^{\prime}=0}\right)\right|_{r=1, \varphi=0} \\
q_{12}=\left.\frac{\partial}{\partial r}\left(\left.\frac{\partial G\left(r, \varphi ; r^{\prime}, \varphi^{\prime} ; E\right)}{\partial r^{\prime}}\right|_{r^{\prime}=1, \varphi^{\prime}=0}\right)\right|_{r=1, \varphi=\pi} .
\end{gathered}
$$

The above mentioned selection rule for the extension leads to the following relations:

$$
\left\{\begin{array}{l}
\alpha_{2}^{j+1}+\alpha_{1}^{j}=\alpha\left(\alpha_{2}^{j} q_{12}+\alpha_{1}^{j} q_{11}\right), \\
\alpha_{2}^{j} q_{12}+\alpha_{1}^{j} q_{11}=\alpha_{1}^{j+1} q_{21}+\alpha_{2}^{j+1} q_{22}
\end{array}\right.
$$

Here, $\alpha$ is the parameter describing the strength of the $\delta$-potential at the contact point. 
Taking into account (5) and (6), we express $a_{j+1}, b_{j+1}$ in terms of $a_{j}, b_{j}$ :

$$
\begin{gathered}
a_{j+1}=\frac{q_{12}}{q_{11}} a_{j}-\frac{1-\alpha q_{11}}{q_{11}} b_{j}, \\
b_{j+1}=a_{j}\left(q_{11}+\frac{\alpha q_{11}-1}{1-\alpha q_{11}}-\frac{q_{11}^{2}-q_{22}^{2}}{q_{11}}\right)+b_{j}\left(\frac{1}{q_{12}}+\frac{\left(1-\alpha q_{11}\right)\left(q_{11}^{2}-q_{22}^{2}\right)}{q_{11} q_{12}}\right) .
\end{gathered}
$$

Using (4), one obtains from (7), (8) the formula for the transfer-matrix $M$ :

$$
M=\left(\begin{array}{cc}
\frac{q_{12}}{q_{11}} & -\frac{1-\alpha q_{11}}{q_{11}} \\
q_{11}-1-\frac{\operatorname{det} Q}{q_{11}} & \frac{1}{q_{12}}+\frac{\left(1-\alpha q_{11}\right) \operatorname{det} Q}{q_{11} q_{12}}
\end{array}\right),
$$

where $\operatorname{det} Q=q_{11}^{2}-q_{12}^{2}$. One can check that $\operatorname{det} M=1$. In order for $E$ to belong to the continuous spectrum of the Hamiltonian, the modula of the eigenvalues of the matrix $M=M(E)$ must be equal to 1 (see, e.g. [14]). In our case, it reduces to the following inequality:

$$
|\operatorname{Tr} M(E)| \leq 2
$$

\section{Results and discussion}

The spectral bands of the Hamiltonian for the periodic chain of circles are obtained by solving equation (2) for various quasi-momenta $q$. The dependence of the dispersion equation solution upon the quasi-momentum is shown in Fig. 2. The picture depends on the value of the magnetic flux $\frac{\Phi}{\Phi_{0}}$. For the semi-integer flux (Fig. 2a), one has only levels (so-called, flat bands, i.e. infinitely degenerate eigenvalues). Fig. $2 b$ shows the band structure for $\frac{\Phi}{\Phi_{0}}=0.2$.
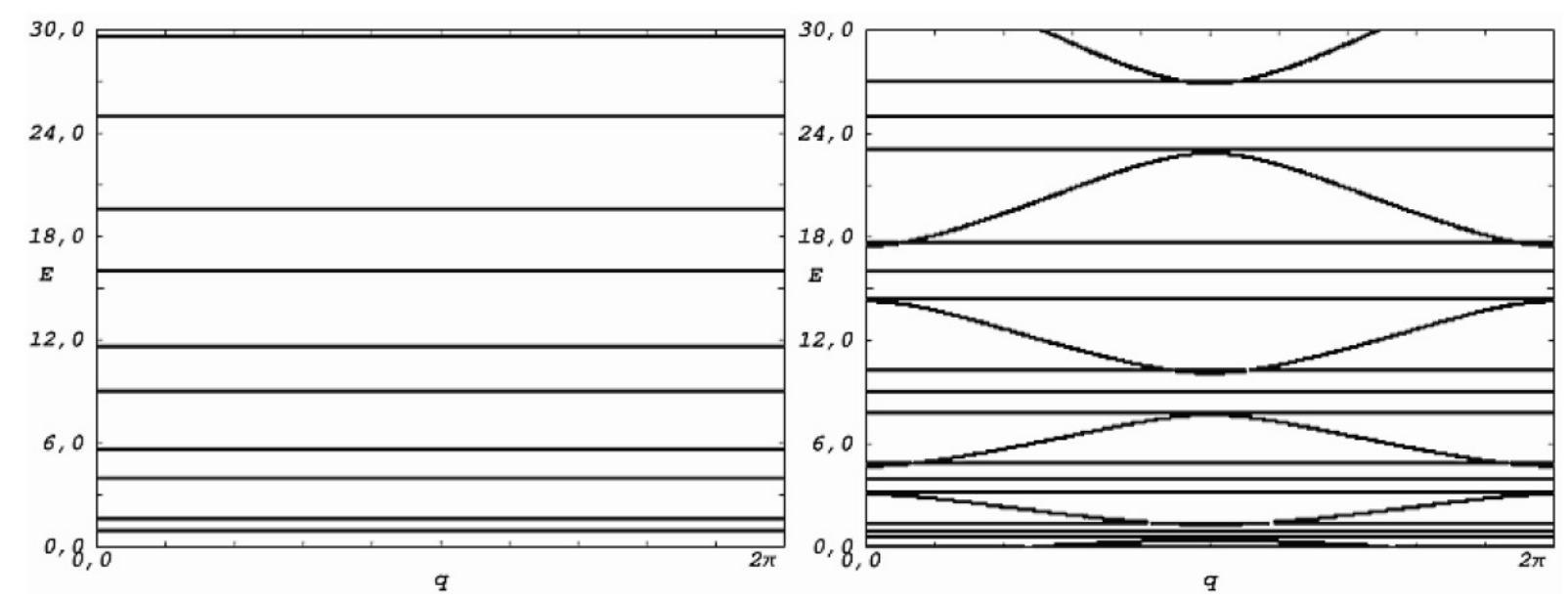

FIG. 2. The band structure for the circles chain for different values of the magnetic flux: $\mathrm{a}-$ for $\frac{\Phi}{\Phi_{0}}=0.5 ; \mathrm{b}-$ for $\frac{\Phi}{\Phi_{0}}=0.2$.

The dependence of the bands on the magnetic field for the disks system for different $\alpha$ parameter values is shown in Fig. 3.

The dependence of the magnetic field bands for both systems is shown in Fig. 4. Bands correspond to the dark areas. One can see that for chosen range of the magnetic field, the lowest band for the chain of circles lies below the lowest band for the chain of disks. Hence, 

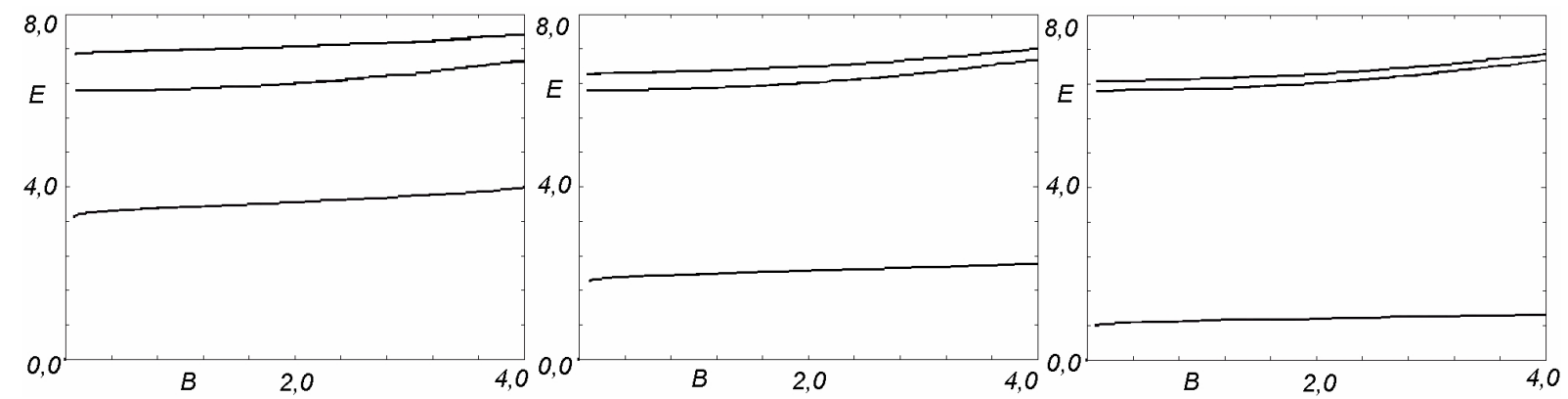

FIG. 3. The dependence of the bands on the magnetic field for the disks system for different $\alpha$ parameter values: $\mathrm{a}$ - for $\alpha=0.5$; $\mathrm{b}-$ for $\alpha=1$; for $\alpha=2$.

for these magnetic field values, one has something similar to the topological insulator effect; the edge state lies below all bulk states.
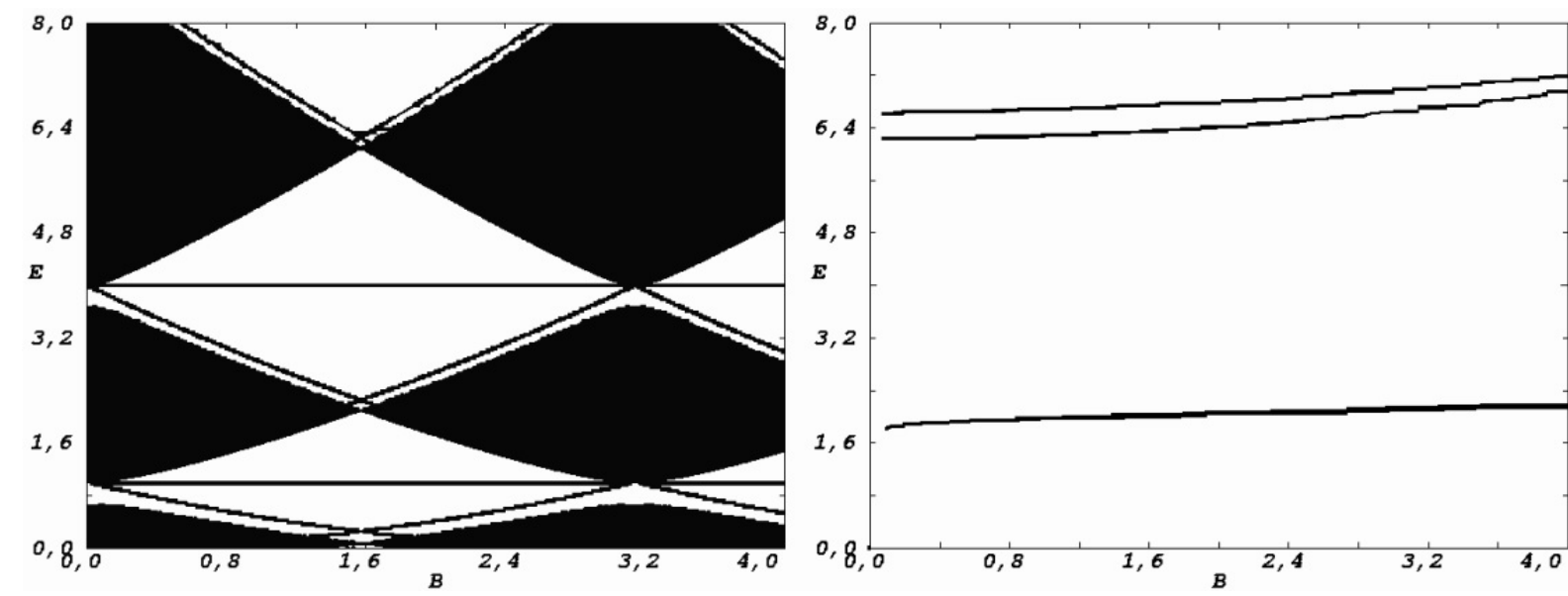

FIG. 4. The dependence of the energy bands on the magnetic field $B$ : a - for the chain of disks; $b$ - for the chain of circles.

\section{Acknowledgements}

This work was partially financially supported by the Government of the Russian Federation (grant 074-U01), by the Ministry of Science and Education of the Russian Federation (GOSZADANIE 2014/190, Project 14.Z50.31.0031), by grants of the President of Russia (state contracts 14.124.13.2045-MK and 14.124.13.1493-MK).

\section{References}

[1] C. Hermosa, J.V. Alvarez, et al. Intrinsic electrical conductivity of nanostructured metal-organic polymer chains. Nature Comm., 2013, 4, P. 1709.

[2] A. Medhi, V.B. Shenoy. Continuum theory of edge states of topological insulators: variational principle and boundary conditions. J. Phys.: Condens. Matter, 2012, 24 (35), P. 355001.

[3] M.Z. Hasan, C.L. Kane. Topological insulators. Rev. Mod. Phys., 2010, 82 P. 3045-3067.

[4] S. Mao, Y. Kuramoto, K.-I. Imura, A. Yamakage. Analytic theory of edge modes in topological insulators. J. Phys. Soc. Japan, 2010, 79, P. 124709.

[5] J.J. Cha, K.J. Koski, Yi. Cui. Topological insulator nanostructures. Phys. Status Solidi RRL, 2013, 7 (1-2), P. $15-25$.

[6] N.I. Gerasimenko, B.S. Pavlov. Scattering problems on noncompact graphs. Theoret. Math. Phys., 1988, 74, P. 230-240. 
[7] P. Exner, P. Seba. Free quantum motion on a branching graph. Rep. Math. Phys., 1989, 28, P. 7-26.

[8] P. Exner, et al. (Eds.) Analysis on Graphs and Its Applications. Proc. Symp. Pure Math., (AMS, Providence, Rhode Island, 2008).

[9] O. Post. Spectral Analysis on Graph-like Spaces. Springer, Berlin, 2010.

[10] I.S. Lobanov, I.Yu. Popov. Scattering by zigzag-armchair nanotube junction. Nanosystems: Physics, Chemistry, Mathematics, 2012, 3 (2), P. 6-28.

[11] I.Yu. Popov, A.N. Skorynina, I.V. Blinova. On the existence of point spectrum for branching strips quantum graph. J. Math. Phys., 2014, 55, P. 033504/1-20.

[12] V. Kostrykin, R. Schrader. Kirchhoff's rule for quantum wires. J. Physics A: Math. Gen., 1999, 32, P. 595-630.

[13] K. Pankrashkin. On the Robin eigenvalues of the Laplacian in the exterior of a convex polygon. Nanosystems: Phys., Chem., Math., 2015, 6 (1), P. 46-56.

[14] P. Duclos, P. Exner, O. Turek. On the spectrum of a bent chain graph. J. Phys. A: Math. Theor., 2008, 41, P. 415206.

[15] I.Yu. Popov, P.I. Smirnov. Spectral problem for branching chain quantum graph. Phys. Lett. A, 2013, 377, P. 439-442.

[16] I.S. Lobanov, E.S. Trifanova. Direct and inverse problems in the model of quantum graphs. Nanosystems: Phys., Chem., Math., 2012, 3 (5), P. 6-32.

[17] I.S. Lobanov, A.I. Trifanov, E.S. Trifanova. Genetic algorithm for constructing graphene nanoribbon with given electronic transport properties. Nanosystems: Phys., Chem., Math., 2013, 4, P. 512-523.

[18] D.A. Eremin, I.Yu. Popov. Quantum ring with wire: a model of two-particles problem. Nanosystems: Phys., Chem., Math., 2012, 2 (2), P. 6-32.

[19] M.A. Kokoreva, V.A. Margulis, M.A. Pyataev. Electron transport in a two-terminal AharonovBohm ring with impurities. Physica E, 2011, 43, P. 1610.

[20] B.S. Pavlov, I.Yu. Popov, V.A. Geyler, O.S. Pershenko. Possible construction of a quantum multiplexer. Europhys. Lett., 2000, 52, P. 196.

[21] I.Yu. Popov. The resonator with narrow slit and the model based on the operator extensions theory. J.Math. Phys., 1992, 33 (11), P. 3794-3801.

[22] I.Yu. Popov. Model of point-like window for electromagnetic Helmholtz resonator. Zeitschrift fur Analysis und ihre Anwendungen. 2013, 32 (2), P. 155-162. 